while the source at R.A. $17^{\mathrm{h}} 44^{\mathrm{m}}$, declination $-28^{\circ} 22^{\prime}$ appears to be less than $1 \cdot 5 \%$ polarized. One would expect strong depolarization effects over most of the galactic centre region.

\title{
Position of the Galactic Centre
}

For the peak of the central source of Figure 1 we find the $1950 \cdot 0$ coordinates:

Right ascension $17 \mathrm{~h} 42^{\mathrm{m}} 30 \mathrm{~s} 0 \pm 2^{\mathrm{s}}$

Declination $\quad-28^{\circ} 58: 7 \pm 0 ! 5$.

This position is a pproximately 4.0 east and 3.7 south of the new IAU coordinates of the galactic centre. A small error may be noted in the positioning of the coordinate grid of Figure 1, which was plotted before the final coordinates were calculated.

\section{Acknowledgments}

We thank Miss Janice A. Milton for assistance in preparing the 10 -cm isophotes, and Mr. T. E. Cousins for setting up the receiver. One of us (R.M.P.) held a Fulbright Fellowship during the course of this work.

\section{References}

Biraud, F., Lequeux, J., and Le Roux, E. (1960).-Observatory 80: 116-18.

Broten, N. W., and Medd, W. J. (1960).-Ap. J. 132 : 279-85.

Drake, F. D. (1959).-N.R.A.O. Annual Report No. 1: 2.

Heeschen, D. S., and Meredith, B. L. (1961).-Publ. N.R.A.O. 1 : 121-8.

Howard, W. E., III, Rood, H. J., and Boyce, P. B. (1962).-Ap. J. 136 : 133-7.

Littue, A. G. (1961).-Stanford Radio Astronomy Inst. Publ. No. 16.

Mathewson, D. S., Healey, J. R., and Rome, J. M. (1962).-Aust. J. Phys. 15 : 354-77.

Mirls, B. Y., Litthe, A. G., Sheridan, K. V., and Slee, O. B. (1958).-Proc. I.R.E. 46: 67-84. Moffet, A. T., and Maltby, P. (1962).-Ap. J. Suppl. $7:$ 93-163.

PariJsky, Y. N. (1959).-Doklady Akad. Nauk SSSR 129: 1261-3; Soviet Phys. Doklady 4 : $1172-4$. Sloanaker, R. M., and Nichols, J. H. (1960).-U.S. Naval Research Laboratory Rep. 5485.

Westerhout, G. (1958).-B.A.N. 14: 215-60.

\section{Discussion}

Davies: Your derivation of the spectral index of the source near the galactic centre using 10 and $3.75 \mathrm{~cm}$ is important. How was the $3.75 \mathrm{~cm}$ brightness temperature calibration of Drake's isophote map derived?

Cooper: I do not know how Dr. Drake calibrated his brightness temperature scale. His map was stated to show isophotes of brightness temperature directly in degrees $\mathrm{K}$. Lequeux says he believes the scale to be in $T_{a}$ rather than in $T_{b}$, thus causing the derived $T_{b}$ to take on a higher value.

\section{THE STRUCTURE OF THE GALACTIC NUCLEUS}

\author{
Y. N. PariJsky \\ Pulkovo Observatory
}

The problem of the nuclei of galaxies is really one of the most important problems on the way to our understanding of numerous very strange phenomena which we observe in our own Galaxy as well as in many others, especially in radio galaxies. 
Accumulating the observational data, we realize more and more that very striking phenomena occur around the excited nucleus of a galaxy. The reason for all these occurrences is quite unknown as yet. That is why the astronomer believes (as the first approximation) that the very singular point of a galaxy, its central region, is the primary source of all unexplained observable features. Thus we can reduce the number of unknown parameters to one only.

The nearest nucleus, that of our own Galaxy, is highly obscured in the optical region and practically invisible. Up to date, we certainly know much more about the nucleus of M31 than about our own. On the contrary, radio observations give very much information about our central region and almost nothing about that of the Andromeda nebula. For this reason we are obliged to combine the optical features for M31 with the radio features for our nucleus, and then to reconstruct the real pattern for the late-spiral type of galaxy. (This must be on the basis of the not very evident analogy between these two galaxies.)

First of all, let us consider the purely observational results which are available in the radio continuum. Many very interesting results have been obtained in the

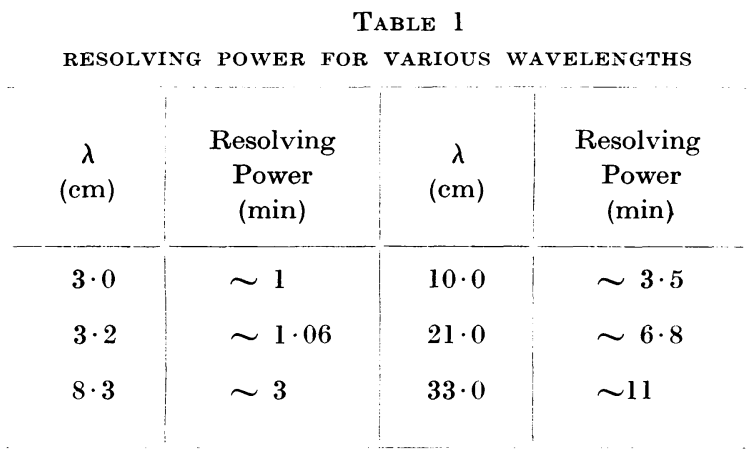

U.S.A. and in France; and we have heard just now some extremely fine Australian results. At Pulkovo, observations of the central region were started early in 1959 at a number of wavelengths (Table 1). All observations were carried out with the help of the large Pulkovo radio telescope.

This telescope is part of an elliptical cone, which converts an incident plane wavefront into a cylindrical one. The second small mirror is a cylindrical paraboloid (now a half-cylindrical paraboloid) which converts a cylindrical wave into a spherical one.

The overall extension of the antenna system is $130 \mathrm{~m}$, and the reflecting surface consists of 90 flat elements, each being $3 \mathrm{~m}$ in height. Observations are possible in a \pm 30 degree range in azimuth, but all the present observations were done exactly in the meridian plane. Careful geodetic measurements were undertaken to get maximum positional accuracy.

Results of the first set of observations are shown in Figure 1.

The following conclusions may be drawn immediately:

(1) There is very sharp detail with an angular diameter $\sim 3^{\prime}$. 
(2) The dependence of the brightness distribution on the frequency is readily seen, and it is difficult to explain this fact by the variation of the resolving power with wavelength. The spectrum of the extended source seems much "steeper" than that of the smaller one.

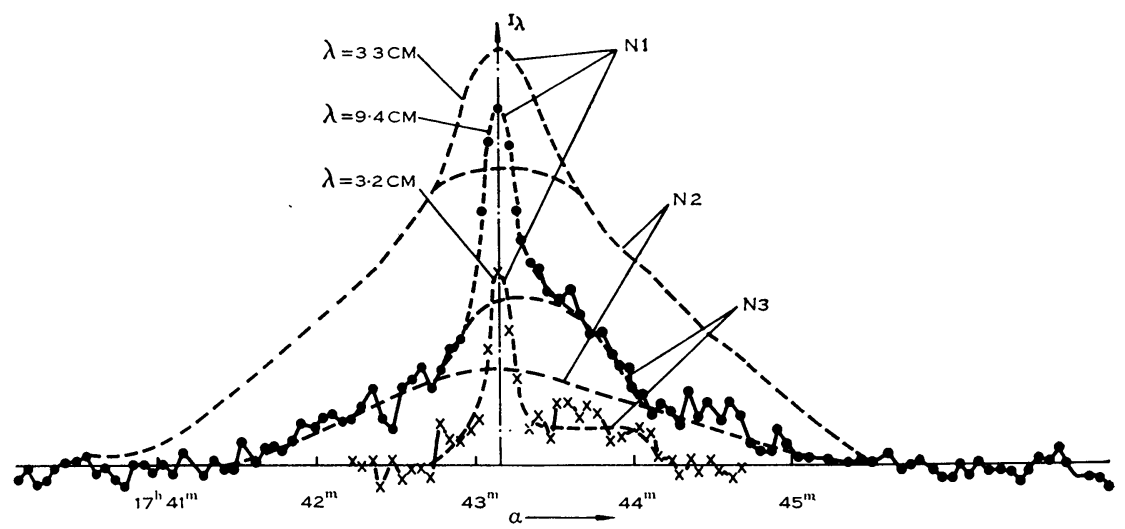

Fig. 1.-Strip scans of the source Sgr A. (Aerial temperature on an arbitrary scale.)

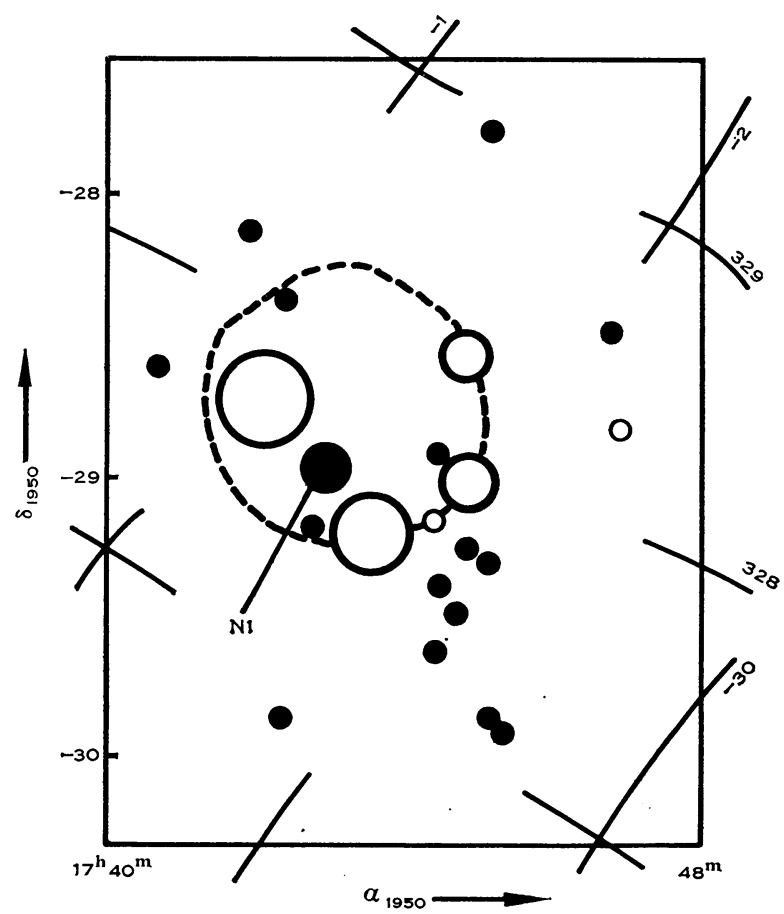

Fig. 2.-The position of the brightest detail (big spot) among the $\mathrm{OB}$ stars (small dots) and emission nebulae (open circles).

(3) The central bright detail is just at the axis of symmetry of the extended source.

(4) The nearby source has disappeared at the longer wavelengths. 
(5) The position of the brightest detail does not coincide either with emission nebulae visible in this direction or with OB stars (Fig. 2). Careful inspection of the Shajn and Gaze plates of this region of the sky brings us to the same conclusion.

The right ascension of this detail (using the Orion nebula as a reference source) is :

$$
\alpha_{1962 \cdot 0}=17^{\mathrm{h}} 43^{\mathrm{m}} 13 \mathrm{~s} 9 \pm 0.7 .
$$

This value may be compared with

$$
\alpha_{1962.0}=17^{\mathrm{h}} 43^{\mathrm{m}} 09^{\mathrm{s}} .0,
$$

the adopted position for the geometrical centre of the Galaxy in the new system of galactic coordinates.

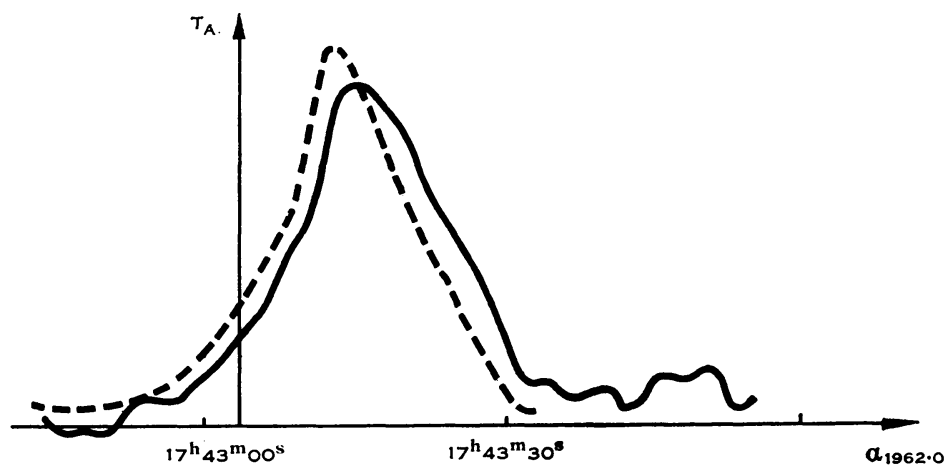

Fig. 3.-Strip scans of the brightest detail of Sgr A at $3 \cdot 2 \mathrm{~cm}$. Full line, observations; broken line, corrected for the time constant.

Thus, judging from the geometry only, it may well be that the extended source and the central detail are really connected with the nucleus of the Galaxy. In 1959 a very clear two-dimensional picture of this region was obtained by Drake. He discovered the following new details:

(1) A spur of emission.

(2) Two sources on either side of the central bright detail.

(3) Faint emission regions, probably associated with emission nebulae.

Again, from the geometrical point of view, Drake believes that these two sources are really a belt of emission.

Now from the geometry we have the following picture: central detail, nonthermal surroundings, and belt of emission. Let us now take the spectrum of the different details into consideration, together with the 21-cm measurements (cf. Ryzhkov et al., this volume, paper 45). We see, first of all, that the $21-\mathrm{cm}$ and geometrical methods give us incompatible results. It seems that the velocity field in this region is extremely complex, and, perhaps, the geometrical and spectrum methods are stronger here. Now let us consider the physical conditions in the two main details-the small and extended sources. We may hope that the central source is at least partially thermal in origin. The physical dimension of this detail is $\sim 10 \mathrm{pc}$ - just the same as in the M31 nucleus. Then we may hope that the distribution of the 
thermal emission (the emission at the shortest wavelength) reflects in some way the distribution of stars in our nucleus. Strong support for this idea is seen in the Orion nebula.

We tried to obtain this distribution last year at $3 \mathrm{~cm}$. The one-dimensional scan of this detail is shown in Figures 3 and 4.

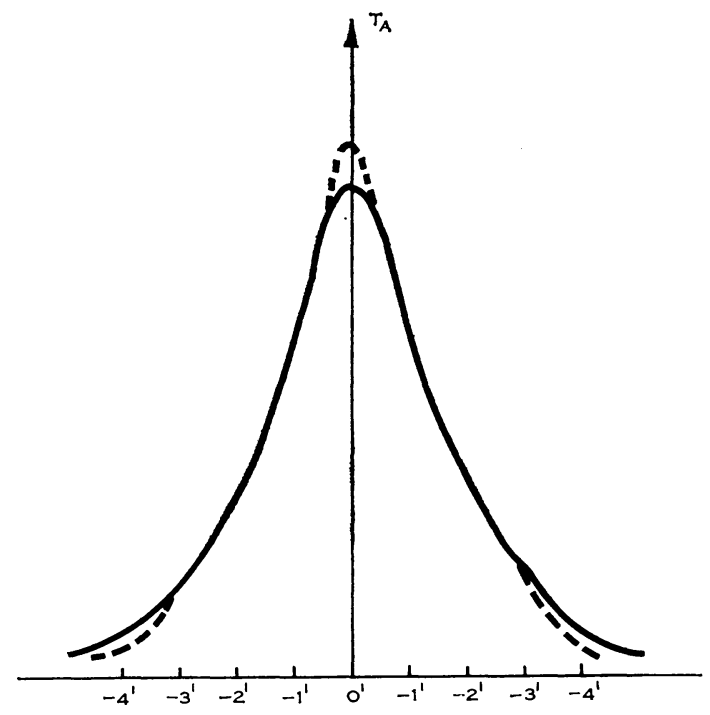

Fig. 4.-Strip scan of the brightest detail of Sgr A at $3 \cdot 2 \mathrm{~cm}$ (smoothed); broken line, corrected for aerial smoothing.

Now we may construct the distribution of emissivity in the very nucleus, provided spherical symmetry is assumed (Fig. 5). It is clear that the accuracy of the observation is not very high, but the very fact of the high concentration at the central point is certainly real. If the mean star density in the nucleus of M31 is $\sim 3 \times 10^{5} \mathrm{pc}^{-3}$, then we may expect that in the small central region of our Galaxy the density of stars is as high as $3 \times 10^{6}$. It seems that $1^{\prime}$ resolution is insufficient to resolve the brightest part of the nucleus.

What may excite gas of such high density? We may calculate that about $1000 \mathrm{OB}$ stars are needed. Another source may be found following the discovery of the new mechanism of dissipation of mechanical into thermal energy. There is an analogy in the Cerenkov emission of magnetohydrodynamic waves behind a star, moving in a plasma with supersonic velocity.

Very interesting conclusions may be drawn analysing the nonthermal source of emission. We can see an energy of $\sim 3 \times 10^{53} \mathrm{erg}$ in the cosmic ray and magnetic field forms. The main losses for these relativistic particles are:

(1) Synchrotron losses: time scale $\sim 10^{6}$ years.

(2) Ionization losses: time scale $\sim 10^{6}$ years.

(3) Free-free transitions: time scale $\sim 10^{6}$ years.

(4) Compton back-scattering: time scale $\sim 10^{5}$ years. 
For comparison, it is interesting to find these losses for a small source:

(1) Synchrotron losses: $\sim 3 \times 10^{5}$ years.

(2) Ionization losses: $\sim 3 \times 10^{4}-3 \times 10^{5}$ years.

(3) Free-free transitions: $\sim 4 \times 10^{4}$ years.

(4) Compton back-scattering: $\sim 10^{2}$ years.

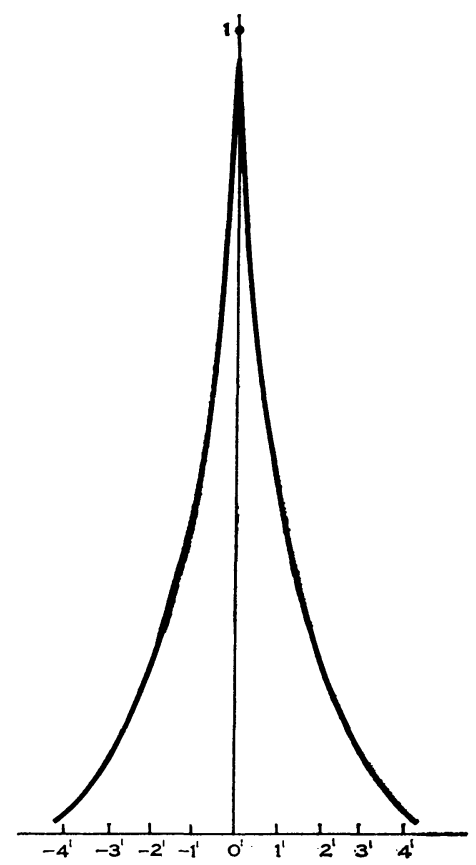

Fig. 5.-Distribution of the emissi-

vity inside the brightest detail.

We therefore come to the conclusion that without a permanent energy supply the source in the centre of the Galaxy must disappear in a time scale $10^{5}-10^{6}$ years. The capacity of this energy supply must be as high as $3 \times 10^{57}-3 \times 10^{58} \mathrm{erg}$. It is easy to show that the usual frequency of supernovae cannot produce this amount, and thus the only known way for the transformation of a star's nuclear energy is insufficient.

The kinetic energy of the stars inside the nonthermal broad source is comparable with this value, and we may find a way for the transformation of this into radio emission, provided the total gas mass ejected from the stars in any way (during the lifetime of the Galaxy) is comparable with the present mass of stars in this region.

We may try to consider the following scheme: (rotational energy of stars) $\rightarrow$ (rotational energy of gas) $\rightarrow$ (magnetic energy) $\rightarrow$ (cosmic ray energy) $\rightarrow$ (radio emission). It is interesting that this scheme must lead to instability of the whole central region and to ejection of the gas and magnetic field with magnetohydrodynamical speed about every $10^{6}-10^{8}$ years. This may be shown by using the virial theorem. 
The attractive point of this scheme is the close connection between all the observable quantities:

(a) star-distribution and motion,

(b) gas-motion (21-cm and optical line emission),

(c) radio emission.

We may try to suggest some kind of circulation of cosmic ray particles - some winds, but we again have an energy difficulty. The nuclear region will burn out all known sources of particles inside our Galaxy. The only way that we have then is to suggest a metagalactic origin of the cosmic rays-metagalactic winds through the nucleus. It is known, however, that the chemical abundance of the cosmic rays does not allow this.

We see that even for our own Galaxy it is very difficult to explain the observable phenomena in the more or less usual way.

I think that it would be very important to work out a concrete international program for studying the nuclei of galaxies, and to have a suitable list of objects to be measured and the quantities to be measured in the first instance. The first step seems to be M31 for radio research, but there are perhaps more suitable objects for these multi-sided studies.

\section{Discussion}

Burke: Do you consider that the cosmic rays come with all their energy from the galactic nucleus, or is it only the injecting source, with the cosmic rays being accelerated later?

Parijsky: The nuclear region differs from the rest of the Galaxy as the time scale for cosmic rays is much smaller in this region. We are at the limit for energy derived from supernovae. The most important step in the processes described with respect to energy conversion in the nucleus is the increase of the magnetic field energy. If this takes place it is not necessary to have very high transformation of magnetic energy as the radio emission rises rapidly with the strength of the magnetic field. Such transformation provides suitable circumstances for an increase of cosmic ray energy. It may well be that in production of the cosmic rays the central source is as powerful as the whole Galaxy.

Burke: In your energy argument you started with the cosmic ray energy density as well as the galactic magnetic energy density. Since in some models the cosmic ray energy density is related to radio emission we should see far greater nonthermal brightness in the central region if the cosmic rays have their origin there.

Lequeux: I am not quite sure that the time scale of the central source of the Galaxy is small. Sources of similar size and emissivity have been observed at Nançay in M31 and M33, and by D. S. Mathewson at Parkes in some other bright galaxies. So the central radio emission is probably at least a recurrent phenomenon.

Parijsky: We may apply observational data, but the statistical data are not available in sufficient quantities as yet. However, we must find what the source of energy is. At present we know no energy source with the time scale needed for the galactic centre. Thus we have two possibilities: (1) there are quite unknown sources of energy, and (2) the radio emission is a recurrent phenomenon.

Kerr: Another indication of the time scales in the nuclear region is given by the expansion velocities in the $3 \mathrm{kpc}$ arm and other features. The indicated times are of the order of tens of millions of years.

Parijsky: We must remember that we cannot extrapolate the expansion velocities to the very central region. One might expect velocities comparable to magnetohydrodynamic velocities and this may reduce the time scale. Also, the 3-kpc arm may have been formed before the central explosion in the nucleus from which we now observe strong radio emission. 
Oort: If one supposes that the expansion velocities observed in the central region are caused by an explosion at the centre, it is necessary to assume that a process of ejection of large amounts of matter from the nucleus has been going on over a long interval of time. While the matter pushing out the $3-\mathrm{kpc}$ arm must have left the nucleus some 50 million years ago, the highvelocity matter with positive velocities observed close to the centre must have started only a few million years ago.

I would like to ask Dr. Parijsky whether he really believes that the emission in the southern part of what he calls a ring is further than $3 \mathrm{kpc}$ from the centre. From the appearance of the structure near the direction of the centre, this seems rather improbable.

Parijsky: As far as the position of the sources is concerned it is indeed very strange if it is not a ring (or another combination of details) in the central region. Of course, it could also be a "window" in the 3-kpc arm.

\section{RECENT OBSERVATIONS AT DWINGELOO OF THE CENTRAL REGION OF THE GALACTIC SYSTEM}

\section{J. H. OoRT}

\section{Leiden Observatory}

The work reported on was done by G. W. Rougoor and is based on observations made with the Dwingeloo dish and the eight-channel receiver built by Professor Muller.

The longitude interval from -8 to $+22^{\circ}$ was studied in detail for four latitudes, at $b^{\mathrm{I}}=-0^{\circ} 5,-1: 0,-1.5$, and $-2^{\circ} 0\left(b^{\mathrm{II}}=\right.$ about $+0^{\circ} 9,+0^{\circ} .4,-0^{\circ} 1$, and $\left.-0^{\circ} 6\right)$. The maximum intensity is in general found at $b^{\mathrm{I}}=-1.5$ (Fig. 1). This level gives the clearest picture of the gas in the small, rapidly rotating disk. Some other features stand out better at other latitude levels. At $b^{\mathrm{I}}=-1.0$ one can trace a rather continuous arm on the positive-velocity side from longitude $22^{\circ}$ down to $-5^{\circ}$. At the point where it passes behind Sgr A this arm appears to have a radial motion of $+135 \mathrm{~km} / \mathrm{sec}^{*}$ Its sudden ending at $l \mathrm{II} \sim-5.5$ suggests that at this point it is close to the outer edge of the disk. But its velocity at this point differs entirely from that of the gas within the disk.

The contour diagram for $b^{\mathrm{I}}=-2^{\circ} .0$ indicates two other features. At the positive-velocity side an "arm" is observed emerging from the general ridge of small velocities around $l \mathrm{II}=12^{\circ}$. The velocity of this arm rises steeply towards smaller longitudes up to a maximum of about $+230 \mathrm{~km} / \mathrm{sec}$ near $l \mathrm{II}=4^{\circ}$, where it may join up with the rapidly rotating disk to be described below. It is possible that this arm could be a continuation of the 3-kpc arm which is such a striking feature on the side of the negative velocities.

The second feature is seen at the negative velocities, crossing the longitude of the galactic centre. At this point it has a velocity of approximately $-100 \mathrm{~km} / \mathrm{sec}$. This may be due either to gas clouds near the edge of the rotating disk and moving away from the centre, or to part of a small expanding arm.

A possible arrangement of the arms and disk in the central region is shown in Figure 4 of paper 1.

*This, as well as all other velocities mentioned, is relative to the local standard of rest. 